\title{
Notiz über den Nierenblutkreislauf.
}

\author{
Von
}

\section{J. Setse he now}

in St. Petersburg.

Hierzu 1 Holzschnitt.

Den Physiologen ist, so viel ich weiss, eine Eigenthümlichkeit in der Anordnung des Blutgefässsystems der Niere entgangen, welche offenbar eine grosse Rolle in der Thätigkeit dieses Organes spielt; - ich meine die Lage der Niere an den grossen Blutbahnen unweit vom Herzen and die relative Ktirze der renalen Gefässe. Derartige Lage haben allerdings noch viele andere Organe und Provinzen des Körpers (das Gefässsystem des Herzens selbst, die Gefässe der Intercostalräume, des Zwerchfells, der Baucheingeweide u. s. w.), wenige von denselben bieten aber einen ebenso kurzen Weg fiir das Blut wie die Niere dar. Insofern ist für den Blutumlauf in der letzteren gerade das Zusammenwirken beider Momente als maassgebend zu betrachten. In der Lage der Niere unweit vom Herzen liegt nnzweifelhaft der Grund fiir einen relativ hohen Druck in der Nierenarterie und relativ niedrigen in der Nierenvene, während die Kürze der Nierengefässe den renalen Blutstrom in eine bedeutende Abhängigkeit von den Blutdruckschwankungen sowohl in der aorta abdomin. als in der v. cava inf. setzt. Zieht man hierbei den Umstand in Betracht, dass die Druckverhältnisse in beiden grossen Gefässen von den Circulationsschwankungen in der Niere selbst so gut wie unabhängig sind, so erhalten die erwähnten topographischen Verhältnisse offenbar die Bedeutung der Hauptregulatoren des renalen Blutumlaufes.

In Anbetracht der grossen Widerstände für die Bewegung des Blutes in der Niere scheint die bedentende Blutdruckdifferenz in dem zu- und dem abführenden Gefässe des Organes zunächst den Sinn einer diese Widerstände compensirenden Vorrichtung zu haben. Die Compensation geht jedoch möglicherweise noch weiter, 
indem dieselbe das Nierenblut trotz der grossen Widerstände relativ rasch fliessen lässt. Letztere Wirkung bedürfte allerdings noch experimenteller Beweise, die erstere ist aber unzweifelhaft.

Die gleichzeitige Regulation des Nierenblutstromes von der Arterie und von der Vene aus erklärt ferner die relative Unempfindlichkeit desselben gegen die Compressionen der Nierenarterie ziemlich einfach. So lange nämlich die Compression keine merkliche Frhöhung des totalen Widerstandes von Seite der Niere veranlasst, gelangt das Blut jenseits der künstlich verengten Stelle mit demselben Energievorrath wie frïher an, und da die Bedingungen des Blutabflusses aus der Niere hierbei unverändert bleiben, so behält anch die den Blutfluss bedingende Druckdifferenz ihre normale Höhe bei. Erst wenn die Compression den totalen Widerstand merklich zu erhöhen beginnt, nimmt die Menge des die Niere speisenden Blutes und die dasselbe durch die Gefässe treibende Druckdifferenz ab.

So lange die Beziehung der Nierengefässe zu der v. cava inf. im Dunkeln blieb, mussten natürlich alle theoretischen Ableitungen der Harnsecretion aus den Blutdruckschwankungen in dem grossen Kreislaufe an gewisser Einseitigkeit leiden. In denjenigen Fällen, wo die Erscheinungen im starken Grade durch die Druckverhältnisse in den Venen mitbedingt sind, mussten die Ableitungen sogar als ungenugend ausfallen. Es gehört hierher nach meiner Meinung das Aufhören der Harnabsonderung nach der Durchschneidung des Ruckenmarks. So lange man die Thatsache auf das Sinken des arteriellen Druckes allein bezieht, ist dieselbe wenig verständlich; so wie man aber noch das gleichzeitige Steigen des Druckes in den Venen, mithin eine bedeutende Abnahme des absoluten Werthes der Druckdifferenz in der art. und v. renalis zu Hülfe ruft, wird die Erscheinung klarer. Am allereinfachsten liess sich aber dieselbe durch die Annahme erklären, dass der mittlere Druck in der v. cava inf. vorzugsweise deshalb steigt, weil die inspiratorische Wirkung des Brustkorbes auf die Venen in Folge der Rückenmarksdurchschneidung wegfällt. Ueberhaupt scheint mir die Frage uber den Einfluss der respiratorischen Bewegungen auf den Blutfluss in der Niere eine experimentelle Bearbeitung zu verdienen.

Ich meinerseits kann einstweilen zu Gunsten des in dieser Notiz Dargelegten nur einige Probeversuche an einem künst- 
lichen Röhrensystem anführen. Hierbei handelte es sich flir mich bauptsächlich um die Entscheidung der Frage, wie gross die Druckschwankungen in dem die untere Hohlvene simulirenden Theile des Systems sein müssen, um merkliche Aenderungen in den Bewegungserscheinungen der Flüssigkeit durch den dem Nierengefässsystem entsprechenden Theil zu geben, vorausgesetzt, dass der totale Widerstand des letzteren dem wirklichen Widerstande der Niere nahezu gleich ist.

Die einfachste diesen Anforderungen entsprechende Röhrencombination ist in der nebenstehenden Zeichnung angegeben. Die Höhe der wassertreibenden Säule $A B$ variirte in den Versuchen zwischen $2-3 \mathrm{~m}$. Die Fortsetzung dieser Säule $B C D$ enthält nebst dem Hahne $G$ ein capillares Rohr $E C F$. Das Röhrenstiick $B E$ entspricht der Bauchaorta; $E C F$ ihren Verzweigungen und das Stiuck $F D$ der unteren Hohlvene. In $m$ zweigt sich von der Bauchaorta das Gefässsystem der Niere in Form einer in horizontaler Ebene hufeisenförmig gebogenen Röhre $m q r$ ab. In dem Stick $m s$, welches dem unverzweigten Theile der Nierenarterie entspricht, befindet sich nebst dem Hahne $n$ ein Quecksilbermanometer $p$; und die Verzweigungen der Nierenarterie sind durch das capillare Rohr $s q r$ dargestellt. Das letztere

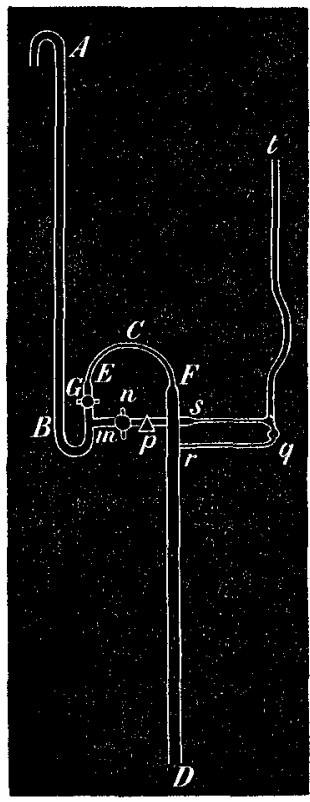
(etwas länger als das Stiick ECF) ist in der Mitte seines Verlaufes etwas aufgeblasen und trägt an diesem Orte ein zum grössten Theil aus Gummirohr bestehendes Wassermanometer $q t$. Unterhalb der Einmündungsstelle der Nierengefässe in die vena cava $(r)$ besteht die letztere ebenfalls aus einem Gummischlauch, so dass man die aspirirende Wirkung der unteren Hohlvene durch Heben oder Senken ihres unteren Endes $D$ leicht reguliren kann. Ich brauche kaum hinzuzuftigen, dass wäbrend das Quecksilbermanometer $p$ der Entscheidung der Frage dient, ob der totale Widerstand der kunstlichen Niere demjenigen der ächten entspricht, das Wassermanometer $q t$ die Druckverhältnisse des capillaren Nierenkreislaufes in ibrer Abhängigkeit ron den 
Druckschwankungen in der Hohlvene anzeigt; und ferner, dass die durch die Niere unter verschiedenem Drucke in der Vene durchgeflossene Wassermenge sich direct bestimmen lässt. Endlich ist noch zu bemerken, dass der Hahn $G$ nicht zur Absperrung des Röhrenstückes $G C F$, sondern auch zur Ausgleichung der Widerstände in diesem Stücke und in der Niere dient.

Versuche an diesem Röhrensystem haben nun folgendes ergeben.

Entspricht der Widerstand der künstlichen Niere demjenigen der ächten und ist der erstere dem Widerstande in dem Röhrenstücke $G C F$ für eine beliebige Höhe der Wassersäule $A B$ (zwischen 2-3 m) gleich gemacht, so wird jede $10 \mathrm{~mm} \mathrm{Hg}$ betragende plötzliche Druckschwankung innerhalb der Hohlvene, sowohl positive als negative, durch eine deutliche Schwankung der Wassersäule in dem Manometer $q t$ beantwortet. Bietet hingegen der Weg durch das Röhrenstlick $G C F$ einen geringeren Widerstand als die Niere dar, so bleibt das Manometer $q t$ nur für positive Druckschwankungen ebenso empfindlich wie vorher, während die aspiratorischen Zuige desto schwächer darauf zu wirken beginnen, je niedriger der Widerstand in GCF sinkt. Letzteres ist ubrigens leicht verständlich. Da die plötzlichen Druekschwankungen in der Hohlvene sich durch die capillaren Theile der Wege GCF und mqr nicht momentan fortpflanzen können, so vertheilt sich zunächst ihre Wirkung in diesen Wegen umgekehrt proportional den Widerständen derselben; aus diesem Grunde müssen die Effecte der Druckschwankungen sich an der Niere iiberhaupt schwächer als an dem anderen durchgängigeren Zweige ausprägen. Ist die Schwankung negativ, so gleicht sich dieselbe an dem Orte ihres Entstehens wegen des vermehrten Wasserzuflusses geschwinder als ein positiver Stoss aus; folglich kann die Wirkung des letzteren an dem widerstandreicheren Zweige noch merklich bleiben, wenn die Effecte der ktirzeren aspiratorischen Zïge schon verschwunden sind.

Der begünstigende Einfluss der Inspirationen auf den Nierenkreislauf scheint hiernach sehr wahrscheinlich zu sein, wenn die Widerstände innerhalb der Leber nicht zu gering im Vergleich mit denjenigen der Niere sind. 